\title{
CORRELATION BETWEEN BALL SPEED OF THE SPIKE AND THE STRENGTH CONDITION OF A PROFESSIONAL WOMEN'S VOLLEYBALL TEAM DURING THE SEASON
}

\author{
David Valadés ${ }^{1}$, José Manuel Palao ${ }^{2}$, Ángel Aúnsolo ${ }^{1}$, and Aurelio Ureña ${ }^{3}$ \\ ${ }^{1}$ Faculty of Medicine and Health Sciences, University of Alcalá, Spain \\ ${ }^{2}$ Department of Health, Exercise Science and Sport Management, \\ University of Wisconsin Parkside, USA \\ ${ }^{3}$ Department of Physical Education and Sport, University of Granada, Spain
}

Original scientific paper

UDC: 796.325-055.2:796.012.1

\begin{abstract}
:
The aim of this paper was to study the relationship between spike speed and the players' characteristics, anthropometrics, and strength levels throughout the season for a women's professional volleyball team. Players from a Spanish first division team performed a battery of tests evaluating anthropometric characteristics, strength performance, and spike speed at the beginning, in the middle, and at the end of one competitive season. The variables were: age; training experience; height; one-hand standing reach height; body mass; body mass index; height of the vertical jump with an approach (spike jump); muscle percentage of arms; 1 repetition maximum (1RM) bench press; 1RM pullover; overhead medicine ball throws for distance using 1 , 2, 3, 4, and $5 \mathrm{~kg}$; spike angles; and speed of standing and jump spikes. Results showed that players' general strength (bench press and pullover) and power parameters (medicine ball throws) increased throughout the season, while speed of the jump spike did not improve. The variable that best predicted the jump spike speed at all the three time points in the season was the standing spike speed. The players' training increased their strength and upper-body power, but these improvements were not transferred to players' hitting speed ability. Push-pull and throwing exercises were not specific enough to improve the hitting ability of the female senior volleyball players.
\end{abstract}

Key words: performance, hitting, test, monitoring, female athletes

\section{Introduction}

The spike is the most effective technical action for scoring in a volleyball rally (Palao, Santos, \& Ureña, 2004; Zhang, 2000). From a mechanical point of view, factors that contribute to the success of a spike are hit height, ball speed, and ball direction (Coleman, Benham, \& Northcott, 1993; Gutiérrez, Ureña, \& Soto, 1994; Kao, Sellens, \& Stevenson, 1994). Given that sport rules stipulate the characteristics of the ball (type, pressure, etc.) and that players have a standardized hand position in the spike, different ball speeds achieved by players are due to their ability to generate speed with their hitting hand (Vint \& Hinrichs, 2004). The hand's hitting speed is determined by the execution of movements of a kinetic chain, which involves the hips, trunk, shoulders, the elbow, and the wrist (Cisar \& Corbelli, 1989; Gutiérrez, et al., 1994; Rokito, Jobe, Pink, Perry, \& Brault, 1998), and it depends on extension velocity in the elbow and shoulder (Chung, Choi, \& Shin, 1990; Ferris, Signorile, \& Caruso, 1995; Singh \& Rathore, 2013), muscle coordination during the kinetic chain performance (Wedaman, Tant, \& Wilkerson, 1988; Masumura, Marquez, \& Ae, 2009), and reach height (Gutiérrez, et al., 1994).

Various studies have assessed the effect of different factors on spike speed performance in volleyball players. Vint and Hinrichs (2004) found, when assessing technique of the female US national volleyball team $2(\mathrm{n}=9)$ using a 3D analysis, that most of the spike speed came from the actions of the elbow $(44.9 \%)$ and shoulder $(30.5 \%)$. Other studies have focused more on the relationship of the spike speed with physical characteristics of players. Among the NCAA Division I female players $(\mathrm{n}=13)$, Ferris et al. (1995) found a negative correlation between the hand's hitting speed and standing reach $(\mathrm{r}=-.576)$ and a positive correlation between the hand's hitting speed and isokinetic upper-body strength in the 
wrist $(\mathrm{r}=.636)$. For male players from the first and second Belgian divisions ( $\mathrm{n}=19)$, Forthomme, Croisier, Ciccarone, Crielaard, and Cloes (2005) found correlations between the hand's hitting speed and hit height ( $\mathrm{r}=.26)$, hours of strength training $(\mathrm{r}=.46)$, isokinetic upper-body strength (flexors and extensors; $\mathrm{r}=.46-.63)$ and external and internal rotators $(\mathrm{r}=-.52-.62)$, and body mass index (BMI) $(\mathrm{r}=.47)$.

All reviewed studies were carried out at a specific moment in the season and none analyzed possible variations of these relationships throughout the season; further, these studies measured the upperbody strength using isokinetic protocols. No studies have been found that have measured upper-body strength using weight training 1RM and medicine ball throws, which are common training methods used by volleyball players for upper-body strength development. The present study also incorporates the use of radar (Ferris, et al., 1995; Forthomme, et al., 2005; Grgantov, Milic., \& Katic 2013; Valadés \& Palao, 2012) in the measurement of the ability to apply force. This strength testing protocol allows us to measure players' general strength, specific strength, and application of strength at different moments of the season (Cook, 2001) and the relationship of these factors with the spike speed. The use of these measurement protocols makes it possible to obtain practical and applicable information to guide volleyball players' strength practice. The analysis of the available information allowed us to hypothesize that aspects most related to jump spike speed were the variables related to the extension speed of the elbow and shoulder, specific muscle coordination, and reach height. The aim of this paper was to study the relationship between spike speed and the players' characteristics, anthropometrics, and strength levels throughout the season for the players from a women's professional volleyball team.

\section{Methods}

Eleven female volleyball players from a team that competed in the Spanish first division voluntarily participated in the study. The mean $( \pm \mathrm{SD})$ age, training experience, body mass, height, BMI, one-hand standing reach height, and jump reach height of the players at the beginning of the study were: $23.82( \pm 5.42)$ years, $11.00( \pm 6.93)$ years, $73.96( \pm 4.89) \mathrm{kg}, 1.82( \pm 0.05) \mathrm{m}, 22.30( \pm 1.13) \mathrm{kg} /$ $\mathrm{m}^{2}, 2.34( \pm 0.06) \mathrm{m}$, and $2.96( \pm 0.08) \mathrm{m}$, respectively. All players included in the study completed all the evaluations. The inclusion criteria for the study were a minimum of five years of training experience (at least two days per week experience in lower- and upper-body strength training using weights and medicine balls) and the absence of shoulder injuries or previous shoulder surgeries. Two players from the team were not included in the study because they did not fulfill the inclusion cri- teria. The Research Ethics Committee of the University of Granada (Spain) pre-approved the study. The subjects received information about the characteristics, procedures, and goals of the study, their voluntary participation and of the possibility to quit at any moment, and confidentiality of the data. The subjects provided written informed consent before the study.

During an 8-week pre-season period, the players trained six days per week (between nine and ten training sessions per week; five in the afternoon and between five and six in the morning). The average training volume was 18.6 hours per week. During the competitive season ( 25 weeks), the players trained five days per week (seven training sessions per week; five in the afternoon and two in the morning) and competed in a weekend match. The average in-season training volume was 11.7 hours per week. During the studied period, the physical conditioning involved a strength circuit performed two days per week with the goal of maintaining specific power and fatigue tolerance levels. The competitive season was divided into three parts (first half of the season, second half of the season, and play-offs). Between the first and second halves of the competitive season, there was a transition period of one week (winter break). Every two weeks, maintenance power training was done ( $3 \times 1$ 1RM for the half squat, pullover, gastrocnemius extensions, and bench press, as well as abdominal and lumbar exercises). During the season's performance peaks, strength was developed by a circuit training that combined strength exercises with technical actions twice a week. The strength circuit included the following exercises: weighted squat jumps, spike jumps, abdominal exercises, lumbar exercises, pullovers, triceps extensions, medicine ball throws to the floor, gastrocnemius extensions, block jumps, quadriceps extensions, hamstring exercises, bench press, and rowing. The circuit was done 3-5 times depending on the moment in the competitive season. For the resistance exercises, athletes completed 6-10 repetitions of $30 \%$ of their $1 \mathrm{RM}$, while for exercises without weights, they carried out movements at a high intensity during 10-15 seconds. Rest time between exercises lasted 30-45 seconds, and between circuits it lasted 3-5 minutes.

The measurements were done at three time points during the season $\left(\mathrm{M}_{1}\right.$, first measurement; $\mathrm{M}_{2}$, second measurement; and $\mathrm{M}_{3}$, third measurement). All the tests were carried out on four consecutive days and each time it was done at least 48 hours after the most recent game. On the first morning of the training week, the maximal strength and power tests were done. On the second and fourth days of the training week, the spike (standing and jump) tests were executed. On the third day of the training week, the anthropometrical measurements were done. On the fourth day of the training week, 
the jump reach tests were done. Between $\mathrm{M}_{1}$ and the mid-season measurement $\left(\mathrm{M}_{2}\right)$, there were eight weeks of training and competitions, and between $M_{2}$ and $M_{3}$ there were five weeks apart. The third measurement was done a week after the team was eliminated from further competition. Before the competitive phase of the season, the team carried out four weeks of pre-season and four weeks of pre-competition work. Before the tests were carried out, the subjects executed standardized warm-ups directed by the primary researcher along with the coach. During all the aforementioned physical tests, the players were verbally encouraged to give their maximal effort.

\section{Measurement of anthropometrical characteristics of the players}

The following anthropometrical measurements were taken in standardized laboratory conditions: body mass (Salter 996, Electronic Scale Batron, USA), body height (Seca 220 measuring rod, Hamburg, Germany), one-hand standing reach height (Seca 206 measuring rod, Hamburg, Germany), and percentage of arm muscle (Skinfold Caliper and Holtain Ltd. flexible metallic tape measure, Pembrokeshire, UK). Further, BMI was calculated as body mass divided by height squared $\left(\mathrm{kg} / \mathrm{m}^{2}\right)$. The arm muscle area was calculated by the perimeter and skinfold. This calculation was done following the criteria described by Heymsfield, McManus, Smith, Stevens, and Nixon (1982). The measurements were taken by a trained researcher (ISAK certification) with more than six years of experience, following ISAK criteria. Anthropometric measures met the requirements of the Technical Error of Measurement (TEM), which were lower than $5 \%$ for skinfolds and lower than $1 \%$ for the other measurements (ISAK, 2001).

\section{Assessing players' strength characteristics}

Strength was assessed in standardized laboratory conditions using the following tests: 1RM bench press $(\mathrm{kg})$; 1RM pullover $(\mathrm{kg})$; overhead distance throws of medicine balls weighing 1,2, 3, 4, and $5 \mathrm{~kg}(\mathrm{~m})$; and height of the vertical jump with an approach (m). All players were familiar with these exercises. A five-minute rest was taken between the bench press test, pullover test, and medicine ball throws. The jump test was completed before the spike test.

- $1 \mathrm{RM}$ bench press $(\mathrm{kg})$ and $1 \mathrm{RM}$ pullover $(\mathrm{kg})$. The maximum weight lifted by the athlete in one repetition was recorded. The measurement protocol used was the one already described in the literature (Chelly, Hermassi, \& Shephard, 2010; Cramer \& Coburn, 2004). A coach helped at the start and end of the trial. As a specific warm-up, two sets of 10 repetitions at $20 \%$ of $1 \mathrm{RM}$ from the previous test were lifted. The highest load with an acceptable extension was registered as each player's 1RM. The recovery period between attempts was two minutes. The players normally took two or three attempts to establish their 1RM. On one occasion, one player took four attempts on her first pullover measurement.

- Overhead medicine ball throws (m). The test consisted of throwing the medicine balls of 1,2 , 3,4 , and $5 \mathrm{~kg}$ overhead as far as possible from a kneeling position that should prevent the effects of lower body on a throw (Viitasalo, 1988). Each player completed three throws with each weight. The rest between sets of three throws was three minutes. The distance reached was measured, and the best trial was used for further analysis. Two coaches monitored the performance of the repetition: one monitored the throw (Viitasalo, 1988), and the other monitored the landing. If the player lost her balance and fell forward, the attempt was discarded.

- Height of vertical jump with an approach (m). The players imitated the approach and spike jump without hitting a ball. The vertical jump height with an approach was measured using a Vertec (Vertec, Questek Corp, Northridge, CA). Then it was calculated by subtracting the one-hand standing reach height from the Vertec measure (Newton, Rogers, Volek, Häkkinen, \& Kraemer, 2006). The better of two trials was used for the analysis. The recovery between trials lasted one minute.

\section{Measurement of standing and jump spikes kinematics}

The ball speed and hitting angle (angle between the hitting arm and the trunk) were measured for the standing and jump spikes. Each player completed five standing spikes and five jump spikes with a 1-minute rest period between the trials. The protocol followed was the one described by Palao and Valadés (2009). The jump spike speed was measured during a specific warm-up. The standing spike speed was measured at the end of a practice in combination with the team's serve training to prevent players from getting cold. The type of ball used was the same as that utilized in all practices and competitions (Molten IV 5XC). The ball pressure was controlled in all practices $(0.30$ to 0.325 $\mathrm{kg} / \mathrm{cm}^{2}$ ). The same trained coach tossed the ball for the jump spike in all evaluations (to three-four meters in height and a half meter from the net). The hitting area was delimited by two antennas (1.5 meters apart). A radar gun (Stalker ATS, Texas, USA) was placed five meters from the net and was protected by a futsal goal (Graph 1). The highest ball velocity $(\mathrm{km} / \mathrm{h})$ for each player was registered. 


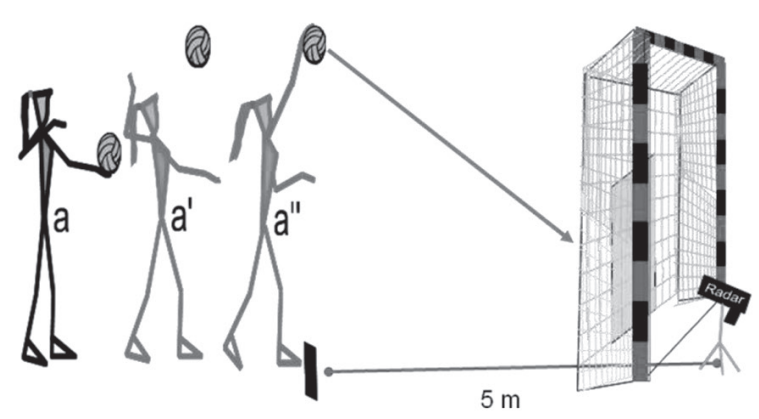

Figure 1. Standing and jump spike evaluation.

All trials were filmed laterally by a digital camera operating at $50 \mathrm{~Hz}$ for later analysis (Sony DCRTRV355E, Digital Hi8, Sony Corp, San Diego, CA).

The camera was mounted on a tripod stand one meter high and six meters sideways from the hitting area. The optical axis of the camera was perpendicular to the direction of the approach, and the field of view of the camera was zoomed so that the athlete was visible in the jumping and hitting phase. Kwon3D biomechanical analysis software (Visol, Cheolsan-dong, Korea) was used to analyze video images of the trial where the highest speed was reached. Twenty-two body landmarks, defining a 14-segment model of the athlete, were digitized in each image. The utilized segmental data were those proposed by De Leva (1996) for adults. The handshoulder angle was calculated as the angle formed by a vector connecting the center of the shoulder joint, the hand point, and the horizontal axis. The hand-hip angle was calculated as the angle formed by a vector connecting the center of the hip joint and the hand point and the horizontal axis (Figure 2). These angles were measured to monitor the biomechanical pattern of spike execution (Gutiérrez, et al., 1994; Vint \& Hinrichs, 2004). Reliability testing of the digitized points used to calculate the angles showed no statistically significant differences in a random jump digitized ten times by a researcher. The intra-class correlation was $>.990$, and the inter-

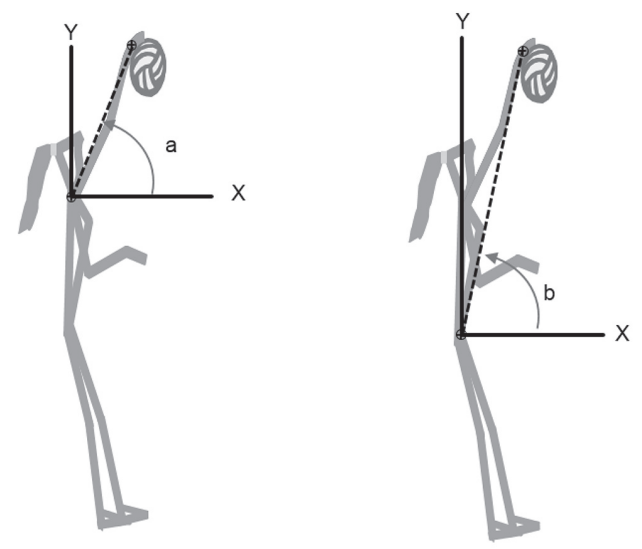

Figure 2. Measurement of the hand-shoulder angle (a) and hand-hip angle (b) in the spike (hit).

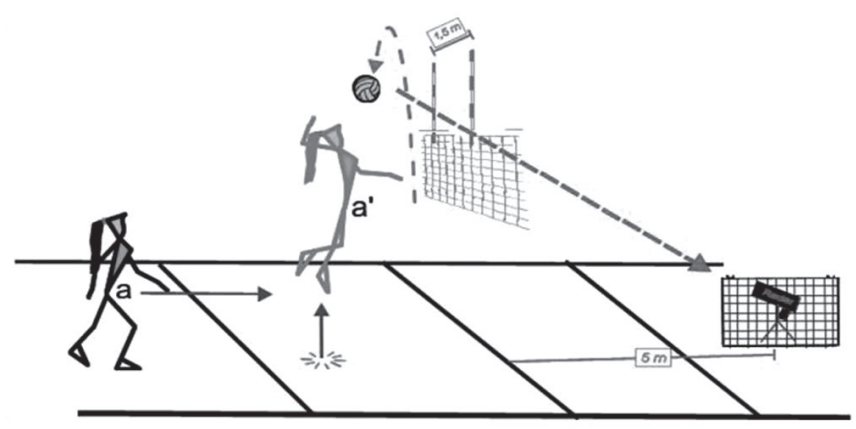

class correlation was $>.970$. The inter-class reliability was calculated using another researcher as a reference. Each of the researchers had more than five years of experience in digitization of sports techniques.

Descriptive (means and standard deviation) and inferential analyses were done. The KolmogorovSmirnov and Shapiro-Wilk's tests were carried out to assess the normality of data distribution. As the data distribution of the sample was non-parametric, the Friedman test and Wilcoxon test were used to compare the results measured and assessed at each time point in the season. A multiple regression was used to verify the association between variables.

\section{Results}

The multiple regression analysis did not show the same predictive model for all the three time points in the season (Table 1). In the first evaluation, the model significantly describing the jump spike speed ( $p=.001$ ), with an $\mathrm{R}^{2}$ of .868, was the standing spike speed (.728) and the vertical jump height with an approach (.426). In the second evaluation, the model significantly predicting the jump spike speed ( $p=.001)$, with an $\mathrm{R}^{2}$ of .870, was the standing spike speed (.652), the vertical jump height with an approach (.887), and body height (-0.397). In the third evaluation, the model significantly describing the spike speed $(\mathrm{p}<.038)$, with an $\mathrm{R}^{2}$ of .459 , was the standing spike speed.

The results did not show any significant change in spike speed (standing or jump); body mass; body height; one-hand height reach; hitting angles (jump spike); or medicine ball throws with 3, 4, or $5 \mathrm{~kg}$ at any stage of testing (Table 2). There were significant increases in the pullover $(8.81 \%, \mathrm{p}<.05)$ and bench press $(5.37 \%, \mathrm{p}<.05)$ weights, as well as in vertical jump height with an approach $(0.52 \%, \mathrm{p}<.05)$, dominant arm muscle area $(7.84 \%, \mathrm{p}<.05)$, and nondominant arm muscle area $(5.82 \%, \mathrm{p}<.05)$ between the first part of the competitive period and midseason $\left(\mathrm{M}_{1}-\mathrm{M}_{2}\right)$. Likewise, there were significant increases in medicine ball throws with $1 \mathrm{~kg}(5.36 \%$, $p<.01)$ and $2 \mathrm{~kg}(4.74 \%, p<.05)$, the pullover $(4.65 \%$, $\mathrm{p}<.05)$ and the bench press $(1.82 \%, \mathrm{p}<.05)$ weights 
Table 1. Multiple regression between jump spike speed and players' characteristics, biomechanical, anthropometric, and strength measurements at three points in the season

\begin{tabular}{|c|c|c|c|c|c|c|c|c|c|}
\hline \multirow{2}{*}{ Variable } & \multicolumn{3}{|c|}{$M_{1}$} & \multicolumn{3}{|c|}{$\mathbf{M}_{2}$} & \multicolumn{3}{|c|}{$\mathbf{M}_{3}$} \\
\hline & $\beta$ & $\mathbf{t}$ & Sig & $\boldsymbol{\beta}$ & $\mathbf{t}$ & Sig & $\beta$ & $\mathbf{t}$ & Sig \\
\hline \multicolumn{10}{|l|}{ Sample characteristics } \\
\hline Age (yr) & 0.16 & 1.19 & 0.27 & -0.09 & -0.92 & 0.39 & -0.05 & -0.18 & 0.86 \\
\hline Experience (yr) & 0.07 & 0.47 & 0.65 & -0.10 & -1.08 & 0.32 & -0.04 & -0.13 & 0.90 \\
\hline \multicolumn{10}{|l|}{ Anthropometrical characteristics } \\
\hline Body mass (kg) & -0.34 & -2.21 & 0.06 & -0.04 & -0.29 & 0.78 & 0.27 & 0.89 & 0.40 \\
\hline Height (m) & -0.13 & -0.75 & 0.48 & -0.40 & -3.17 & 0.02 & 0.28 & 1.00 & 0.35 \\
\hline BMI $\left(\mathrm{kg} / \mathrm{m}^{2}\right)$ & -0.15 & -1.11 & 0.31 & -0.03 & -0.27 & 0.80 & 0.01 & 0.03 & 0.97 \\
\hline One-hand reach height $(\mathrm{m})$ & 0.09 & 0.42 & 0.69 & 0.10 & 0.48 & 0.64 & 0.36 & 1.37 & 0.21 \\
\hline Dominant arm muscle area $\left(\mathrm{cm}^{2}\right)$ & -0.04 & -0.27 & 0.80 & -0.06 & -0.67 & 0.53 & -0.16 & -0.59 & 0.58 \\
\hline Non-dominant arm muscle area $\left(\mathrm{cm}^{2}\right)$ & -0.03 & -0.18 & 0.86 & -0.07 & -0.71 & 0.50 & -0.12 & -0.45 & 0.67 \\
\hline \multicolumn{10}{|l|}{ Players' strength characteristics } \\
\hline Pullover 1RM (kg) & 0.12 & 0.79 & 0.45 & -0.17 & -1.01 & 0.35 & 0.07 & 0.23 & 0.82 \\
\hline Bench press 1RM (kg) & 0.01 & 0.06 & 0.96 & 0.07 & 0.65 & 0.54 & 0.22 & 0.81 & 0.45 \\
\hline $1 \mathrm{~kg}$ medicine ball throw $(\mathrm{m})$ & 0.11 & 0.68 & 0.52 & -0.01 & -0.04 & 0.97 & 0.07 & 0.23 & 0.83 \\
\hline $2 \mathrm{~kg}$ medicine ball throw $(\mathrm{m})$ & 0.03 & 0.15 & 0.89 & 0.03 & 0.23 & 0.82 & 0.12 & 0.38 & 0.72 \\
\hline $3 \mathrm{~kg}$ medicine ball throw $(\mathrm{m})$ & 0.06 & 0.28 & 0.79 & -0.01 & -0.10 & 0.93 & 0.15 & 0.48 & 0.65 \\
\hline $4 \mathrm{~kg}$ medicine ball throw $(\mathrm{m})$ & 0.05 & 0.23 & 0.83 & 0.00 & 0.00 & 1.00 & 0.12 & 0.38 & 0.72 \\
\hline $5 \mathrm{~kg}$ medicine ball throw $(\mathrm{m})$ & 0.09 & 0.48 & 0.65 & -0.02 & -0.17 & 0.87 & 0.16 & 0.49 & 0.64 \\
\hline Vertical jump test with approach (m) & 0.43 & 3.20 & 0.01 & 0.89 & 7.18 & 0.00 & 0.35 & 1.43 & 0.20 \\
\hline \multicolumn{10}{|l|}{ Standing and jump spike kinematics } \\
\hline Standing spike $(\mathrm{km} / \mathrm{h})$ & 0.73 & 5.48 & 0.00 & 0.65 & 7.29 & 0.00 & 0.68 & 2.60 & 0.03 \\
\hline Hand-shoulder angle $\left({ }^{\circ}\right)$ & 0.12 & 0.83 & 0.44 & -0.15 & -1.32 & 0.23 & -0.22 & -0.74 & 0.48 \\
\hline Hand-hip angle $\left({ }^{\circ}\right)$ & -0.17 & -0.96 & 0.37 & -0.16 & -1.18 & 0.28 & -0.16 & -0.55 & 0.60 \\
\hline
\end{tabular}

Note: $\mathrm{M}_{1}$ - measurement taken in the first part of the competitive season; $\mathrm{M}_{2}$ - measurement taken mid-season; $\mathrm{M}_{3}$ - measurement taken at the end of the competitive season.

Table 2. Ball speed, biomechanical, anthropometric, and strength measurements at the three points in the season

\begin{tabular}{|c|c|c|c|c|c|c|}
\hline \multirow{2}{*}{ Variables } & \multirow{2}{*}{$\begin{array}{c}M_{1} \\
\bar{X} \pm S D\end{array}$} & \multirow{2}{*}{$\begin{array}{c}M_{2} \\
\bar{X} \pm S D \\
\end{array}$} & \multirow{2}{*}{$\begin{array}{c}M_{3} \\
\bar{X} \pm S D\end{array}$} & \multirow{2}{*}{$\begin{array}{c}\text { Friedman } \\
\qquad \mathrm{p}\end{array}$} & \multicolumn{2}{|c|}{ Wilcoxon } \\
\hline & & & & & $M_{1}-M_{2}$ & $M_{2}-M_{3}$ \\
\hline \multicolumn{7}{|l|}{ Anthropometrical characteristics } \\
\hline Body mass (kg) & $73.96 \pm 4.89$ & $73.43 \pm 5.30$ & $73.45 \pm 5.04$ & 0.18 & $-0.73 \%$ & $0.02 \%$ \\
\hline Height (m) & $1.82 \pm 0.05$ & $1.82 \pm 0.05$ & $1.82 \pm 0.05$ & . & $0.00 \%$ & $0.00 \%$ \\
\hline BMI $\left(\mathrm{kg} / \mathrm{m}^{2}\right)$ & $22.30 \pm 1.14$ & $22.14 \pm 1.28$ & $22.15 \pm 1.23$ & 0.18 & $-0.73 \%$ & $0.04 \%$ \\
\hline One-hand reach height (m) & $2.34 \pm 0.06$ & $2.34 \pm 0.06$ & $2.34 \pm 0.06$ & . & $0.00 \%$ & $0.00 \%$ \\
\hline Dominant arm muscle area $\left(\mathrm{cm}^{2}\right)$ & $42.32 \pm 7.97$ & $45.92 \pm 8.49$ & $45.25 \pm 8.99$ & $0.01^{*}$ & $7.84 \%{ }^{*}$ & $-0.37 \%$ \\
\hline Non-dominant arm muscle area $\left(\mathrm{cm}^{2}\right)$ & $42.54 \pm 7.67$ & $44.73 \pm 9.25$ & $43.87 \pm 9.54$ & 0.06 & $5.82 \% *$ & $-3.00 \%$ \\
\hline \multicolumn{7}{|l|}{ Players' strength characteristics } \\
\hline Pullover 1RM (kg) & $27.86 \pm 4.43$ & $30.32 \pm 3.70$ & $31.73 \pm 2.79$ & $0.01^{* *}$ & $8.81 \% *$ & $4.65 \% *$ \\
\hline Bench press 1RM (kg) & $47.36 \pm 6.45$ & $49.91 \pm 7.06$ & $50.82 \pm 6.91$ & $0.00^{* *}$ & $5.37 \%{ }^{*}$ & $1.82 \% *$ \\
\hline $1 \mathrm{~kg}$ medicine ball throw $(\mathrm{m})$ & $10.66 \pm 1.72$ & $10.59 \pm 1.83$ & $11.16 \pm 1.77$ & $0.01^{* *}$ & $-0.64 \%$ & $5.36 \% * *$ \\
\hline 2 kg medicine ball throw (m) & $8.11 \pm 1.37$ & $8.16 \pm 1.30$ & $8.55 \pm 1.25$ & $0.04^{*}$ & $0.56 \%$ & $4.74 \% *$ \\
\hline $3 \mathrm{~kg}$ medicine ball throw $(\mathrm{m})$ & $6.59 \pm 1.09$ & $6.48 \pm 1.10$ & $6.70 \pm 1.32$ & 0.25 & $-1.72 \%$ & $3.51 \%$ \\
\hline 4 kg medicine ball throw $(m)$ & $5.45 \pm 0.80$ & $5.43 \pm 0.86$ & $5.52 \pm 0.94$ & 0.44 & $-0.42 \%$ & $1.67 \%$ \\
\hline $5 \mathrm{~kg}$ medicine ball throw $(\mathrm{m})$ & $4.89 \pm 0.68$ & $4.86 \pm 0.71$ & $4.91 \pm 0.85$ & 0.84 & $-0.47 \%$ & $0.93 \%$ \\
\hline Vertical jump test with approach (m) & $2.97 \pm 0.08$ & $2.98 \pm 0.09$ & $2.97 \pm 0.08$ & $0.04^{*}$ & $0.52 \%{ }^{*}$ & $-0.27 \%$ \\
\hline \multicolumn{7}{|l|}{ Standing and jump spikes kinematics } \\
\hline Jump spike speed (km/h) & $76.36 \pm 4.11$ & $77.00 \pm 5.14$ & $77.27 \pm 5.88$ & 0.54 & $0.83 \%$ & $0.35 \%$ \\
\hline Standing hit speed $(\mathrm{km} / \mathrm{h})$ & $74.36 \pm 5.64$ & $75.18 \pm 6.08$ & $75.27 \pm 5.59$ & 0.14 & $1.10 \%$ & $0.12 \%$ \\
\hline Hand-shoulder angle $\left({ }^{\circ}\right)$ & $74.04 \pm 7.74$ & $79.53 \pm 9.91$ & $76.90 \pm 7.92$ & 0.20 & $7.40 \%$ & $-3.31 \%$ \\
\hline Hand-hip angle $\left({ }^{\circ}\right)$ & $78.32 \pm 4.58$ & $81.18 \pm 7.97$ & $80.20 \pm 5.44$ & 0.67 & $3.64 \%$ & $-1.20 \%$ \\
\hline
\end{tabular}

Note: $M_{1}$ - measurement taken in the first part of the competitive season; $M_{2}$ - measurement taken mid-season; $M_{3^{--}}$measurement taken at the end of the competitive season.

$M_{1}-M_{2} \& M_{2}-M_{3}$, percentage of increase between the measurement points in the season.

${ }^{*}$ Level of significance $p<.05$; ** Level of significance $p<.01$ (Friedman test and Wilcoxon test). 
between mid-season and the last part of the competitive period $\left(\mathrm{M}_{2}-\mathrm{M}_{3}\right)$.

\section{Discussion and conclusions}

The aim of this paper was to study relationships between the spike speed and the players' characteristics, anthropometrics, and strength levels throughout the season for a women's professional volleyball team. The data analysis revealed two aspects in relation to the variables that affected jump spike speed. First, the model that best described the spike speed changed throughout the season. The variables involved in the model were related to the players' ability to apply strength, their standing spike speed, and vertical jump height with an approach. Second, in the examined sample the jump spike speed did not improve, although general strength (bench press and pullover) and power (medicine ball throws) increased throughout the season.

The model that described the spike speed did not include any of the general strength and power variables that were measured. It only included variables related to players' ability to apply strength (standing spike speed, in all three measurements, and the vertical jump with an approach, in $\mathrm{M}_{1}$ and $\mathrm{M}_{2}$ ). These results indicate the importance of motion specificity in proper evaluation of elite athletes. Therefore, due to the similar muscles involved, the execution angles, temporal aspects, etc., standing spike can be used as a test to monitor players' hitting ability in lab conditions or when there is no possibility to test jump spike (Palao, et al., 2004; Palao \& Valades, 2012) with no increases in jump load put on the players (Bahr \& Bahr, 2014; Reeser, Verhagen, Briner, Askeland, \& Bahr, 2006). Otherwise, jump spike should be the test due to its specificity. Standing spike may be also used to work on the hitting ability and complement the upper-body strength training to develop specific power.

In the second measurement, body height negatively influenced the jump spike speed. The reasoning for the specific effect of this variable in the second measurement for this sample is not clear. Ferris et al. (1995) found a negative correlation between jump spike speed and standing reach heights for female NCAA Division I players. The authors hypothesized that the tallest players have better spike angles and they do not need to hit at the highest speed to be successful in the game.

Forthomme et al. (2005) found a relationship between the players' jump ability and the spike speed for male players of different competitive levels (Division I $=0.78 \mathrm{~m}$ over the net, Division II $=0.62 \mathrm{~m}$ over the net), which could explain a lower spike speed of players at lower competitive levels. When jump height is low, it can affect the spike speed once the net becomes a high obstacle to be overcome in the attempt to hit the ball towards the target zone. However, the players' vertical jump height with an approach in the present study $(0.73 \mathrm{~m}$ over the net by female players) was high enough to be discarded as an aspect that hindered their attack angle or the time players had to execute the spikes.

Data collected on the upper-body general strength and power showed improvement for the players studied. However, the improvement did not result in an increase in the jump spike speed, standing spike speed, or hitting angles. Similar improvements in the players' general and specific strength ability were found by Marques, Van den Tillaar, Vescovi, and González-Badillo (2008), Gadeken (1999), and Piper (1997), although these studies measured players' ability to lift weights (e.g. pullovers and bench press) and to throw an apparatus (e.g. medicine ball), not the ability to impart speed to the ball. This finding suggests that combining weight lifting with medicine ball throws may not have been specific enough to transfer strength gains to the spike speed. For the lower body, training resulted in a negligible increase in the players' vertical jump with an approach $(0.01 \mathrm{~m})$.

Another possibility behind this lack of improvement found in spike speed is that the players' power work, using push-pull exercises and medicine ball throws, does not involve enough speed in the shoulder and elbow extension moves or in the performance of a movement as a whole. Additionally, these movements are not specific enough (e.g. bilateral vs. unilateral, no trunk rotation, no hip flexion, push vs. hit, performed on the floor vs. performed in the air) for the volleyball spike motion. The players' training improved their ability to move and throw light loads but not their ability to hit the ball faster. Hitting exercises with a light ball, such as the one used in the game $(0.260-0.280 \mathrm{~kg})$, were not done. Also, it must be taken into consideration that when players hit the ball, they rotate the trunk and flex the hips (Gutiérrez, et al., 1994); critical motions that were not present in the training that was carried out. These findings suggest that it may be necessary to review specific strength and power training for volleyball to ensure transfer of the gains obtained to the ability to impart speed to the ball.

The results showed importance of training specificity in the development of elite athletes' actions since general upper-body strength and power did not improve spike speed of the elite female volleyball players studied. This lack of transfer found in our data reveals a need for more investigations on the relations of upper-body strength training and spike speed in elite volleyball players. For a proper contextualization of the findings, several aspects of the study must be considered as delimitations and limitations of the study. Limitations included the criteria used in the study of the technique (e.g. camera's low sampling rate, variables analyzed) and protocols to measure the upper-body strength (e.g. overhead throwing the ball for distance from 
a kneeling position). Future studies on this subject should specifically focus on how to improve spike speed by applying physical training that better imitate the spike motion (e.g. airborne medicine ball throws) along with kinematic analysis.

The model that best described the spike speed included the most specific actions studied: the standing spike speed and jump ability. These find- ings reinforce importance of specificity in training and monitoring elite athletes. Improvements in the players' general strength and upper-body power did not transfer to the spike speed, indicating that the use of push-pull exercises and throwing activities from a kneeling position are not specific or adequate enough to create proper stimuli in terms of motion velocity and form of execution.

\section{References}

Bahr, M.A., \& Bahr, R. (2014). Jump frequency may contribute to risk of jumper's knee: A study of inter-individual and sex differences in a total of 11943 jumps video recorded during training and matches in young elite volleyball players. British Journal of Sports Medicine, 48, 1322-1326.

Chelly, M.S., Hermassi, S., \& Shephard, R.J. (2010). Relationships between power and strength of the upper and lower limb muscles and throwing velocity in male handball players. Journal of Strength and Conditioning Research, 24(6), 1480-1487.

Chung, C., Choi, K., \& Shin, I. (1990). Three dimensional kinematics of the striking arm during the volleyball spike. Korean Journal of Sports Sciences, 2, 124-151.

Cisar, C.J., \& Corbelli, J. (1989). The volleyball spike: A kinesiological and physiological analysis with recommendations for skill development and conditioning programs. NSCA Journal, 11(1), 4-9.

Coleman, S., Benham, A.S., \& Northcott, S.R. (1993). A three-dimensional cinematographical analysis of the volleyball spike. Journal of Sports Science, 11, 295-302.

Cook, G. (2001). Baseline sports-fitness testing. In B. Foran (Ed.), High performance sports conditioning (pp.19-48). Champaign, IL: Human Kinetics.

Cramer, J.T., \& Coburn, J.W. (2004). Fitness testing protocols and norms. In R. Earle \& T. Baechle (Eds.), NSCA's essentials of personal training. Chapter 11 (pp. 235). Champaign, IL: Human Kinetics.

De Leva, P. (1996). Adjustments to Zatsiorsky-Seluyanov's segment inertia parameter. Journal of Biomechanics, 29(9), 1223-1230.

Ferris, D., Signorile, J.F., \& Caruso, J.F. (1995). The relationship between physical and physiological variables and volleyball spiking velocity. Journal of Strength and Conditioning Research, 9(1), 32-36.

Forthomme, B., Croisier, J.L., Ciccarone, G., Crielaard, J.M., \& Cloes, M. (2005). Factors correlated with volleyball spike velocity. American Journal of Sports Medicine, 33(10), 1513-1519.

Gadeken, S.B. (1999). Off-season strength, power, and plyometric training for Kansas State volleyball. Strength and Conditioning Journal, 21(5), 49-55.

Grgantov, Z., Milic. M., \& Katic, R. (2013). Identification of explosive power factors as predictors of player quality in young female volleyball players. Collegium Antropologicum, 37(Suppl. 2), 61-68.

Gutiérrez, M., Ureña, A., \& Soto, V. (1994). Biomechanical analysis of the hit in the volleyball spike. Journal of Human Movement Studies, 26, 35-49.

Heymsfield, S.B., McManus, C., Smith, J., Stevens, V., \& Nixon, D. (1982). Anthropometric measurement of muscle mass: Revised equations for calculating bone-free arm muscle area. American Journal of Clinical Nutrition, 36, 680-690.

ISAK. (2001). ISAK international standards for anthropometric assessment. Adelaide: International Society for the Advancement of Kinanthropometry.

Kao, S., Sellens, R.W., \& Stevenson, J.M. (1994). A mathematical model for the trajectory of a spiked volleyball and its coaching application. Journal of Applied Biomechanics, 10, 95-109.

Marques, M.C., Van den Tillaar, R., Vescovi, J.D., \& González-Badillo, J.J. (2008). Changes in strength and power performance in elite senior female professional volleyball players during the in-season: A case study. Journal of Strength and Conditioning Research, 22(4), 1147-1155.

Masumura, M., Marquez, W.Q., \& Ae, M. (2009). Front and pipe spikes in female elite volleyball players: Implications for the improvement of pipe spike techniques. In Proceedings of XXVI Symposium of the International Society of Biomechanics in Sport (pp. 723-726). Limerick: ISBS.

Newton, R.U., Rogers, R.A., Volek, J.S., Häkkinen, K., \& Kraemer, W.J. (2006). Four weeks of optimal load ballistic resistance training at the end of season attenuates declining jump performance of women volleyball players. Journal of Strength and Conditioning Research, 20(4), 955-961.

Palao, J.M., Santos, J.A., \& Ureña, A. (2004). Effect of team level on skill performance in volleyball. International Journal of Performance Analysis in Sport, 4(2), 50-60. 
Palao, J.M., \& Valadés, D. (2009). Testing protocol for monitoring spike and serve speed in volleyball. Strength and Conditioning Journal, 31(6), 47-51.

Palao, J.M., \& Valadés, D. (2012). Validity of the standing spike test as a monitoring protocol for female volleyball players. Biology of Sport, 29(4), 35-38.

Piper, T.J. (1997). In-season strength / power mesocycle for women's collegiate volleyball. Strength and Conditioning Journal, 19(3), 21-25.

Reeser, J.C., Verhagen, E.A.L.M., Briner, W.W., Askeland, T.I., \& Bahr, R. (2006). Strategies for the prevention of volleyball related injuries. British Journal of Sports Medicine, 40(7), 594-600.

Rokito, A.S., Jobe, F.W., Pink, M.M., Perry, J., \& Brault, J. (1998). Electromyographic analysis of the shoulder function during the volleyball serve and spike. Journal of Shoulder and Elbow Surgery, 7(3), 256-63.

Singh, A.B., \& Rathore, V.S. (2013). Kinematic factors of off-speed and power spike techniques in volleyball. Journal of Education and Practice, 4(7), 112-117.

Valadés, D., \& Palao, J.M. (2012). The radar as an instrument to monitor training. Kronos, 11(1), 30-35.

Viitasalo, J. (1998). Evaluation of explosive strength for young and adult athletes. Research Quarterly for Exercise and Sport, 59(1), 27-28.

Vint, P.F., \& Hinrichs, R.N. (2004). Factors related to the development of ball speed and to the incidence of one-legged landings in the front-row volleyball attack. In Proceedings of the XXII Symposium of the International Society of Biomechanics in Sport (pp. 135-138). Ottawa: ISBS.

Wedaman, R,. Tant, C., \& Wilkerson, J. (1988). Segmental coordination and temporal structure of the volleyball spike. In Proceedings of the VI Symposium of the International Society of Biomechanics in Sport (pp. 577586). Bozeman, MT: ISBS.

Zhang, R. (2000). How to profit by the new rules. The Coach, 1(1), 9-10.

Submitted: October 10, 2014

Accepted: April 24, 2016

Correspondence to:

David Valadés Cerrato

Faculty of Medicine and Health Sciences,

University of Alcalá

Campus Científico-Tecnológico, Ctra. Madrid-

Barcelona Km 33,600.

28871 Alcalá de Henares, Madrid

Phone: + 34918852519

Fax: + 34918854855

E-mail: david.valades@uah.es 